Revista Herencia Vol. 28 (2), 35-42, 2015

Recibido 17-08-2015 Aprobado 22-09-2015

\title{
Arquitecturas de lo Sensible: INSCRIPCIONES URBANAS EN LA CIUDAD UNIVERSITARIA
}

\begin{abstract}
Resumen
En este ensayo se avanza un argumento sobre la dimensión territorial de las inscripciones urbanas mediante la comprensión de las superficies arquitectónicas como artefactos que cumplen funciones estratégicas y usos tácticos. De este modo, este trabajo analiza temas de territorialidad humana y la capacidad social para producir espacios significativos. El ensayo se basa en una deriva realizada por el campus universitario y su entorno.
\end{abstract}

Palabras claves: ciudad; deriva; graffiti; inscripciones urbanas; psicogeografía; territorio.

\begin{abstract}
This essay advances an argument on the territorial dimension of urban inscriptions by understanding surfaces as architectural artefacts that are subject to both strategic and tactical uses. In doing so, this paper discusses issues on human territoriality and social capacity for territory-making. The article is based on a derive made across the campus and its surroundings.
\end{abstract}

Keywords: city; dérive; graffiti; urban inscriptions; psychogeography; territory.

Ana Paula

Montes. Arquitecta (2003) por la Universidad de Costa Rica (UCR) Y Máster en Urbanismo (2010) por la Universidad Politécnica de Cataluña, España (UPC). Actualmente trabaja como docente en la Escuela de Arquitectura y Urbanismo del Instituto Tecnológico de Costa Rica, en la Escuela de Arte y Comunicación Visual de la Universidad Nacional y en la Escuela de Arquitectura de la Universidad de Costa Rica. anapaula.montes@gmail.

\section{Introducción a la escritura visceral y catártica}

Es "abajo" al contrario (down), a partir del punto donde termina la visibilidad, donde viven los practicantes ordinarios de la ciudad. Como forma elemental de esta experiencia, son caminantes, Wandersmänner, cuyo cuerpo obedece a los trazos gruesos y a los más finos [de la caligrafía] de un "texto" urbano que escriben sin poder leerlo. Estos practicantes manejan espacios que no se ven; tienen un conocimiento tan ciego como en el cuerpo a cuerpo amoroso. Los caminos que se responden en este entrelazamiento, poesía inconsciente de las que cada cuerpo es un elemento firmado por muchos otros, escapan a la legibilidad. Todo ocurre como si una ceguera caracterizara las prácticas organizadoras de la ciudad habitada. Las redes de estas escrituras que avanzan y se cruzan componen una historia múltiple, sin autor ni espectador, formada por fragmentos de trayectorias y alteraciones de espacios: en relación con las representaciones, esta historia sigue siendo diferente, cada día, sin fin. (De Certeau, 1980) 


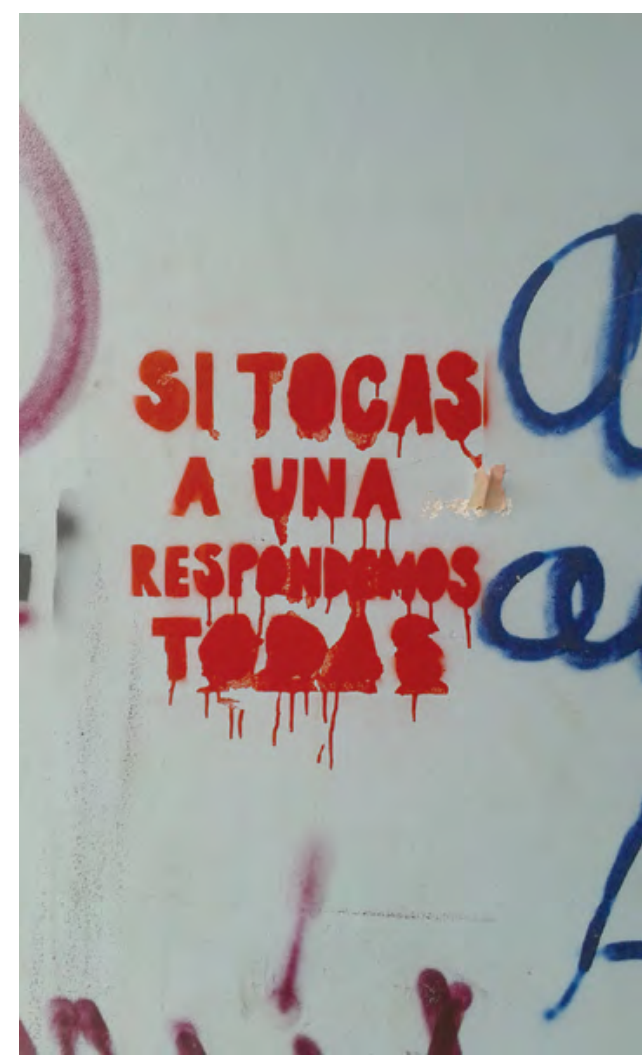

"Si tocas a una respondemos todas", "(In) justo no diga que yo lo provoqué" son algunos de los mensajes que se pueden encontrar cada día caminando por los rincones la Ciudad Universitaria Rodrigo Facio en San Pedro de Montes de Oca. Se trata de inscripciones urbanas que se manifiestan abierta y directamente en las paredes, el mobiliario y cualquier superficie arquitectónica. Emergen de los trazos anónimos hechos con marcador, boligrafo, tiza o lo que tenga a mano el usuario del campus en el momento de inspiración.

Suld hace cuanto se quedaron los baños de humanidades $\sin$ algo que decir"... el apego a los muros parlantes 0 fue por de ponerlos a narrar elocuente y perspicazmente, fue por mucho tiempo sello identitario de la apropiación de los espacios y resquicios del campus de la UCR. Estos mensade los estudiantes con una realidad social que prova de los estudiantes con una realidad social que provocaba desazon, pero que, como telon de fondo, enmarcar a para a estas inscripciones urbanas, el campus de la UCR emería como un ambiente situacionista cuyos rasgos emia serían lo efímero, lo cambiante, lo azaroso y lo indeterminado. En las grafías de la población universitaria estabnipoder de remodelar o intervenir las superficies arquitectónicas, casi a su antojo a manera de juego personal.

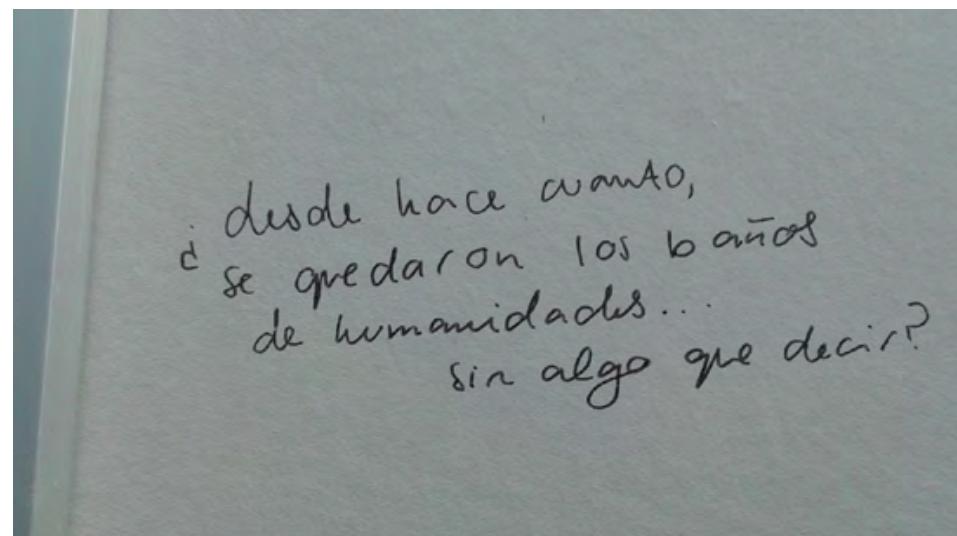

No obstante una reciente deriva No obstante una reciente deriva un silenciamiento de muchos de estos mensajes descorazonadamos de estos rales y catárticos. Ante esta situación rales $\mathrm{y}$ catárticos. Ante esta situación que este escrito pone en valor el papel de tacto entre dos escalas, la arquitectónica y la urbanística. nica y la urbanística.

Este articulo rastrea, en el primer apartado, la manera en la que las inscripciones urbanas configuran un espacio arquitectonico, en tanto que
muestran un uso político y cultural;

Foto 1. Julio 2015, en el segundo apartado, se observa que entrelazados articulan un espacio urbano,

Foto 1. Julio 2015,
Campus Universitario Rodrigo Facio, antiguo edificio Facultad Ciencias Sociales.

en tanto que referencial y cartográfico.

De tal forma que las inscripciones urbanas, no disponen de un empleo exclusivo en el ámbito de la arquitectura, al contrario éstas evidencias ponen en entre dicho que el espacio arquitectónico sea aquel espacio que solamente un profesional de la arquitectura crea y reivindican así el vínculo entre el edificio y el entorno que le rodea. quitectura crea y reivindican asi el vinculo entre el edificio y el entorno que le rodea. certeza es mucho más que una respuesta correcta y eficiente ante una necesidad de uso y se abre la vía de la comprensión de la arquitectura como posibilidad y potencia. Justamente en el tercer apartado se hace referencia a esta capacidad del practicante del espacio para producir e inscribir significados e historias múltiples desde su experiencia directa con lo arquitectónico y urbano.
Dimensión arquitectónica de las inscripciones urbanas

Dijimos antes que las inscripciones urbanas poseen una dimensión arquitectónica, puesto que su naturaleza textual está vinculada a una materialidad que se desempeña tanto estrateg como tácticamente.

En su dimensión estratégica, las superficies arquitectónicas operan como dispositivos de concrol, cierre o separaciôn, mientras que las inscrip-

apropiación del espacio: se suceden en los resquicios, en las espacialidades olvidadas, l margen de lo legal, en la penumbra, de forma fugaz o efímera. (Mubi, 2008)

Las superficies arquitectónicas (paredes, muros, pavimentos, mobiliario, entre otros) constituyen límites estables (Lynch, 1960) que definen una materialidad física del espacio, que orienta flujos y tránsitos, impone conductas y restringe o condiciona Certea 1980) que re finen constantemente los significados simbólicos del espacio. "Hay un pota en la ciudad".. El carácter táctico de las inscripciones urbanas está n su natura en su naturaleza visible: al dejar una huella, desplegar trazas, marcas, firmas que se to de azanción para un determinado público, definen nuevas formas de interacción social, bajo otras reglas y códigos. Asi, los usuar reglas y códigos.

A "l, los usuarios/graffiteros no son personajes pasivos ni resignados que reciben "superficie" tal cual la dotó el arquitecto / diseñador, sino personajes activos que reaccionan ante lo dado - diseñado, ofreciendo resistencia mediante la apropiación creativa, furtiva, selectiva y temporal.

Una suerte de resistencia, dice De Certeau (De Certeau, 1980), que no es directa contra el poder, sino subrepticia, opaca, ambigua, según la cual dicho poder (el poy alterado en su significado por aquellos practicantes del espacio que de forma cotidiana disputan la creación de significados e historias. Coincidiendo con la teoría lefebvriana de la producción del espacio, según la cual, entran en juego tres esferas, la física, la representacional y la experiencial'; tanto el muro como la ciudad como el mismo campus universitario se podrían comprender bajo la idea de "collage" como un acto de reescritura y superposición constante de relatos que revelan los múltiples usos y significados que tienen los espacios que se habitan y ocupan.

En este sentido, podría decirse que estas inscripciones urbanas, historias mínimas sobre temas e intereses variados, contadas por múltiples autores anónimos, son/ fueron un recurso narrativo de la arquitectura, un recurso que revelaba justamente la complejidad de un espacio que era al mismo tiempo una práctica espacial, la representación del espacio y el espacio de representación. Precisamente, esta ciudad collage, obliga ser abordada desde otras herramientas de aproximación y estudio, y una de ellas es la perspectiva psicogeográfica ${ }^{3}$ de la ciudad y los espacios.

"Revolución pelicortas"... hoy se palpa en las paredes y muros de la "U" una extraña textura, la rugosidad de las abyectas paredes rayadas de los baños del edificio de Estudios Generales han sido sustituidas por una higiénica y pulida epidermis acrílica. "Fuerza y $(r)$ evolución" las paredes de la universidad solían gritar a todo pulmón no sólo en clave de protesta sobre los dilemas sociales y políticos del país; también 


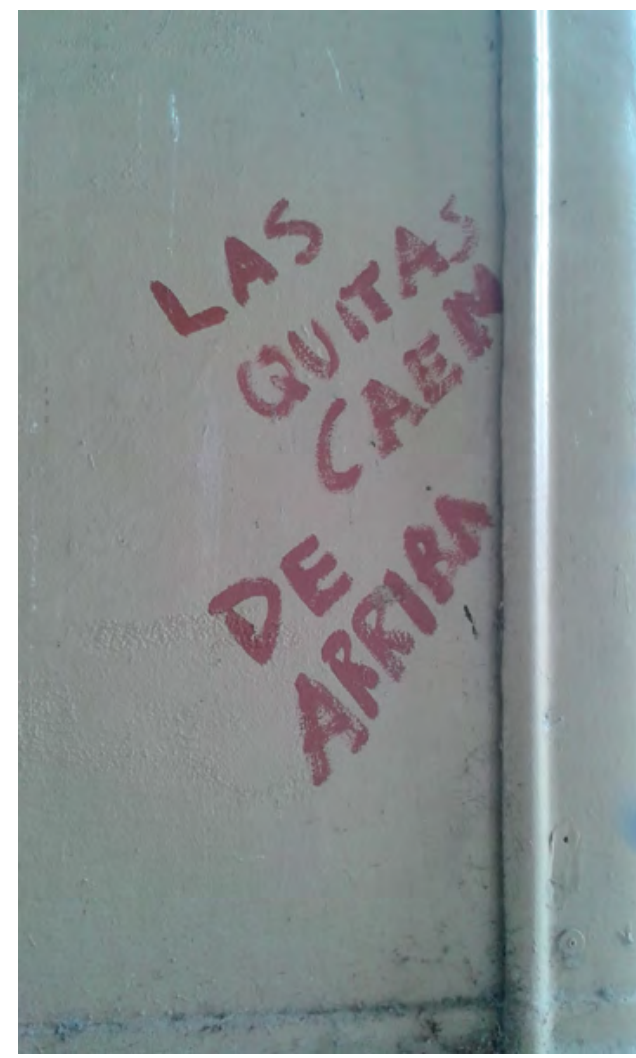

confesaban con frecuencia, historias personales, afectos, conflictos y repudios. Porque, como bien dice Pérgolis, no hay narrativa que no reconozca a la ciudad como el espacio existencial (Pergolis \& Moreno, 2013) en el que convergen lugares, recorridos, actores sociales y acontecimientos. ¿Pero que dicen o decian las inscripciones urbanas de la arquitectura? Narraban una práctica espacial, unas maneras de apropiar y ocupar la arquitectura por parte de la población cives dan una respuesta sión", es decir, reven re tancias decir, revelan reglas del espacio, ordenes y circunsagenciamientos tácticos regidos por el lugar pero también por el "momento oportuno", con lo cul, las inscripcion urbanas son a ta como io explica Michas ins una práctica del tiz, como lo expión (griego, kairos), es un una practica del tienpo. La ocasión (griego, karros), es un produce una ruptura instaura practicas y su extrañanas, porque ble una transgresión de la ley del lugar La ocasión, propicia una "jugada" con poder de modificar el orden loca propicia posible una transformación visible por medio de recursos invisibles: trazos anónimos, disimulados, mínimos).

Así, explica De Certeau, el tiempo Asl, explica De Cécán por completo una situación, implica la implanta cambia memoria en un lugar $y$ hace de la memoria el medio de transformar los espacios (De Certeau, 1980). Pero qué se podría especular en torno a una merma de muros rayados con relación la identidad e historia de la $U$ considerando que explica Pergolis:

"En un diálogo permanente con sus usuarios, la ciudad se convierte en objeto para la realización de múltiples deseos; son imborrables, son huellas en el inconsciente de cada uno de sus usuarios y en el colectivo de la ciudad son su memoria. (Pérgolis \& Moreno, 2013). Un recuerdo: "Daniel... Daniel.."

\section{Dimensión urbana de las inscripciones urbanas}

Pero también, el carácter táctico de las inscripciones urbanas reverbera en una escala más amplia, está vinculado a su naturaleza territorial, en tanto que la arquitectura es constituyente del espacio urbano; las inscripciones urbanas se sitúan en la convergencia de fuerzas que debaten entre lo público, lo privado, lo común y lo personal.

Comprender las inscripciones urbanas como una escritura emocional, una especie de marca espacio - temporal que narran las relaciones y los afectos que se establecen entre los actores sociales y los espacios que habitan; supone comprender el papel de las inscripciones urbanas en la producción de arquitectura de proximidad. En este sentido los mensajes desplegados constituyen un espacio íntimo y más cercano para quien lo recorre o habita. Esta condición de proximidad, familiaridad e intimidad, en el sentido de contacto e interacción en el espacio, es clave para que pueda emerger una cultura de apropiación de espacios comunes, que puede finalmente devenir en una suerte de cartografías sensibles, o puntos de referencia que se comparte con complicidad. Así, las inscripciones urbanas de un modo $\mathrm{u}$ otro dibujan unos mapas de afecto, donde cada usuario establece un recorrido guiado por la práctica de escritura - lectura del espacio que apropia. Estos puntos de referencia para la navegación social del espacio, conforman cartografias reconocibles para las y los actores sociales y por tanto, más útiles en contraposición a las desigun mapa de ubicacin del campus universtario por ms un mapa de ubicacion del campus universitario.

作 ros de acción poltica dentro de la ciudad mismos que son ios de accion politica dentro de la ciudad, mismos que son partir de designaciones oficiales de la propia administración "Estado laco ya." "No al Estado confesional " el registro

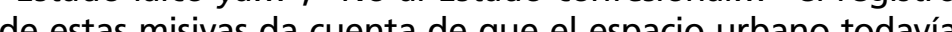
es un lugar de convocatoria colectiva, de reivindicación tavia toriat, un espacio para creer que la transformación es posible. Las inscripciones urbanas narran otras ciudades y otros Lasios, aquellos que nunca aparecerán en las oficiales guías turísticas. Los espacios, paredes y superficies que las gulas turisticas. Los espacios, parios y superficies que las fuerzas de una maquinaria de urbanización genérica (Koolhaas, 2005) aséptica, y autista. has, 2005) aséptica, y autista.

Las inscripciones urbanas podrían entenderse como una exeriencia topo-sensible (Chmielewska, 2008) que da cuenta de as dimensiones temporales - históricas, político - espaciales de 列 nudos, acumulan los múltiples significados y circunstancias del contexto en el que se inscriben.

\section{Lo urbano más allá de la arquitectura}

Investigar las inscripciones urbanas supone adentrarse en los procesos de producción del espacio procesos de producción del espacio simbolico, (posible) que un espacio arquitectónico (certero), sino por los usos tácticos un espacio arquitectónico (certero), sino por los usos tácticos que se suceden justamente en la intersección entre actores sociales (practicantes) y los espacios que apropia. Esta apropiación del espacio es táctica, justamente porque toman por diseñados para decir o contar nada.

Comprender que las inscripciones urbanas replican en el plano de lo afectivo (táctico) pero también lo hacen en el plano arquitectónico (estratégico), supone entender que, justamente en la experiencia con el espacio - narrado, es donde el espacio arquitectónico trasciende la simple forma física contenida entre muros para devenir espacio simbólico. Precisamente lo
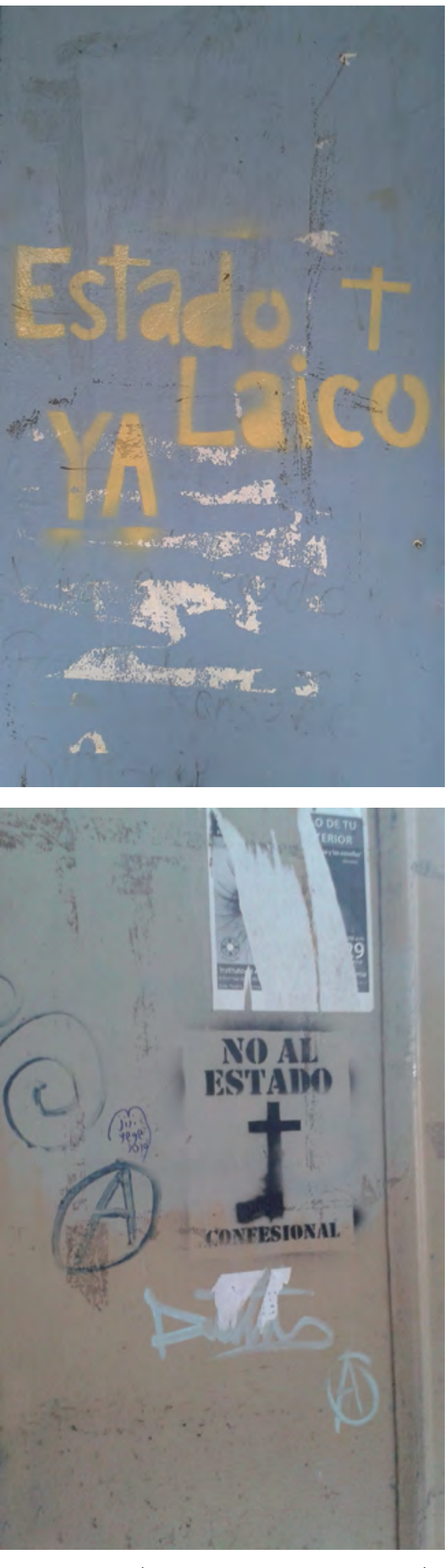

Foto 6 y 7. Julio 2015, Campus Universitario Rodrgo Facio, antiguo edificio Facultad Ciencias Sociales. 
- 1 urbano sucede más allá de lo estrictamente arquitectónico cuando este espacio - narrado emerge de ciertas arquitecturas que operan como ejercicios de comunicación visual y de ciertas inscripciones urbanas que operan como arquitecturas 5 .

Como todo espacio social, el espacio urbano resulta de un determinado sistema de relaciones sociales [...] una proliferación de marañas relacionales compuesta de usos, componendas, impostaciones, rectificaciones y adecuaciones mutuas [...] es escenario y producto haciéndose a sí mismo [...] es una mera actividad, una acción interminable cuyos protagonistas son esos usuarios que inderpre an la forna urbana [...] El espacio urbano no es el resultado de una determichá mor fología predispuesta por el proyecto urbanistico sino de una dialéctica inanternmpidamente re(Delgado, 2007)

das inscripciones urbanas como agenciamientos tácticos dan cuenta de la perspectiva del derecho a práctica del derecho a la ciudad, desde la perspectiva del derecho a acceder a los espacios, transformarlos sugiere que la arquitectura no concluye como tarea, por el hecho de culminar qua obra de infraestructura formalmente llamativa o sobre de culpor la materialidad de sus superficies, al contrario, tanto más importa po (in) tangible, (in) formal, (i) legal precisamente, todas las relaciones

Si la vida urbana subyace sobre los sucesos de la cotidianidad, sobre las prácticas, costumbres y usos tácticos que ocurren de forma efímera cies, más que en la identidad formal el espacio entre los volúmenes y superfFoto 8. Julio 2015. Se podría decir que una arquitectura de lo sensible reacciona y asume conscientemente

la necesidad de propiciar relaciones fluidas y ritmos sociales que son clave para que la vida urbana ocurra.

mundo verifica de las tecnologías de información y comunicación TIC's, donde el munín vistencia virtualmente, las inscripciones urbanas, en contraposiexperiencia topo - sensible. Convierten a los muros en un tación de fuerzas sociales antagónicas en el proceso de creación de significados e identidades.

El hacker urbano $0^{6}$ no es un especialista en tecnologías de información y comunicacion, sino un practicante del espacio, cualquier actor y actriz social, paseante usuario que premeditadamente marca señales y deja trazas para designar espacios significativos dentro de la ciudad cautiva de la maquinaria global de urbanización que homogeniza, estandariza, deshumaniza el espacio social.

"Me gustaría que hubiera lugares estables, inmóviles, intangibles, intocados y casi inTecales, inmutables, arraigados; lugares que fueran referencias, puntos de partida, principios: Mi país natal, la cuna de mi familia, la casa donde habría nacido, el árbol que habría visto crecer (que mi padre habría plantado el día de mi nacimiento), el desván de mi infancia lleno de recuerdos intactos.

Tales lugares no existen, y como no existen el espacio se vuelve pregunta, deja de ser evidencia, deja de estar incorporado, deja de estar apropiado. El espacio es una duda: continuamente necesito marcarlo, designarlo; nunca es mío, nunca me es dado, tengo que conquistarlo. (Perec, 2001).

\section{Notas}

Los miembros de la Internacional Situasionista definen el concepto de deriva de la siguiente manera, mientras Debord hace referencia a la dérive como una forma de crear itinerarios completamente nuevos e impredecibles, dependientes de la casualidad y de los impulsos y reacciones espontáneas y subjetivas. (Debord, 1999); Khatib explica que la deriva es un comportamiento experimental en la sociedad urbana, un modo de acción y un medio de conocimiento dispuesto conscientemente o no, para comprender cómo
espacio interviene directamente sobre el comportamiento afectivo. (Khatib, 1958).

2. Lefebure se plantea la superación del concepto de espacio como absoluto, ópticogeométrico, euclidiano-cartesiano-newtoniano, puesto el espacio no podía concebirse como aislado, vacio o estático, el concepto exigla una profundización de las nociones de producción. Propone una teoria del espacio que liga lo mental y lo cultural, lo socialy lo historico de la cuna se deriva la

3. La psicogeografía, estudio de las leyes y efectos concretos del medio geográfico sobre el comportamiento gráficas e intelectuales dándoles mayor complejidad, enriqueciéndolas. (Khatib, 1958).

4. Para Martí Perán, otras maneras de hacer mapas supone apostar por unas cartografías esquizoanalíticas con poder de deconstruir, la cartografía mediada por los medios. (Perán, 2013)

5. En la Guía Psicogeográfica de París de 1957, Guy Debord realiza un mapa sobre las acciones amorosas que reinterpreta la planta de París y que se obtiene de un procedimiento organización del territorio en función de cómo se organiza la movilidad los núcleos productivos y habitacionales un mapa básicamente atravesado por una pulsión afectiva. En los últimos a nos es notable el desarrollo de tácticas de escritura que buscan reconquistar el espacio como superficie diáógica. En este sentido una instalación en el espacio público de Perejaume titulada Allo que devem estar dibuixant amb les nostres formes de viure en español Aquello que estaremos dibujando con nuestras formas de vida, interpela al transénte para que desconfie de los mapas y se convierta en su propio cartóngrafo. En PERÁN M. (2013) "Maneras de Hacer Mapas". Revista de la Escuela de Arquitectura de la Universidad de Costa Rica. Vol 2-2013. Número 4. p. 105-122.

6. Traducción del término "space hackers" aquel que practica el "space hacking", la práctica de contar nuevas historias sobre la ciudad desco pida, gracias a la cual sería posible una de contar nuevas historias sobre la ciudad desconocida, gracias a la cual sería posible una Pile S. (2001) The un (known) city... or, an urban geography of what lies buried below the surface" publicado en Borden, I.: Kerr, J.; Rendell, J.; Pivaro, A. (eds). The Unknown City: Contesting Architectural and Social Space. Cambridge, MA: MIT Press. P.262-278.

\section{Referencias}

Libros

Debord, Guy. (1999) Internacional Situacionista, vol. I: La realización del Arte. Madrid: Literatura Gris. 
Delgado, Manuel. (2007) Sociedades Movedizas. Pasos hacia una antropología de las calles. Barcelona: Anagrama.

Khatib, Abdelhafid. (1958) "Intento de Descripción Psicogeográfica de Les Halles". Texto aparecido en el \# 2 de Internationale Situationniste. Traducción extraída de Internacional situacionista, vol. I: La realización del arte, Madrid, Literatura Gris, 1999.

Koolhaas, Rem. (2006) La Ciudad Genérica. Barcelona: Gustavo Gili.

Lefebvre, Henri. (1974) La Production de L'Espace. Paris, Éditions Anthropos. En español: Lefebvre, H. (2013) La producción del espacio. Madrid: Capitán Swing. Introducción y traducción de Emilio Martínez.

Lynch, Kevin. (1960) The Image of the City. Cambridge Massachussettes: MIT Press.

Perec, Georges (2001) Especies de Espacios. Barcelona: Montesinos.

\section{Artículos publicados en libros colectivos}

Pile, Steve (2001). The Un (known) City... or, an urban geography of what lies buried below the surface. In: Borden, lain; Kerr, Joe; Rendell, Jane and Pivaro, Alicia eds. The Unknown City: Contesting architecture and social space. Cambridge, Mass.: MIT Press, pp. 262-279.

\section{Publicaciones periódicas}

Perán, Martí. (2013) "Maneras de Hacer Mapas". Revista de la Escuela de Arquitectura de la Universidad de Costa Rica. Vol 2-2013. Número 4. p. 105-122.

Pérgolis, Juan Carlos; Moreno Hernández, Danilo. (2013) "Espacio Público: Narrativas y Deseos". Revista Nodo N 14, Vol. 7, Año 7: 21-34 Enero-Junio. P.21-34

\section{Documentos consultados en Internet}

Chmielewska, Ella (2008) "The wall as Witness-Surface or, the Reichstag Graffiti and Paradoxes of Writing Over History". En: Lo quaderno 08 Usi dei muri / Uses of walls. Disponible en: http://www.losquaderno.professionaldreamers.net/ Consulta: 22 de julio del 2015 año.

De Certeau, Michel. (1980) L'Invention du Quotidien. Vol. 1, Arts de Faire. En español: (2000) La Invención de lo Cotidiano 1. Artes de Hacer; traducido por Alejandro Pescador. México DF, México: ITESO, Universidad Iberoamericana. Capítulo VII, Andar en la ciudad. Disponible en: http://www.bifurcaciones.cl/007/reserva.htm\#2 Consulta: 22 de julio del 2015.

Mubi Brighenti, Andrea. (2008) "The Wall and The City". En: Lo quaderno 08 Usi dei muri / Uses of walls. Disponible en: http://www.losquaderno.professionaldreamers.net/ Consulta: 22 de julio del 2015. 